\title{
Memoria de trabajo y habilidades matemáticas en estudiantes de educación básica
}

\author{
Working memory and mathematical skills in elementary school students
}

Memória de traballho e competências matemáticas nos alunos do ensino primário

\author{
Cesar Augusto Hernández-Suárez ${ }^{1}$ \\ Juliana Paola Méndez-Umaña² \\ Luis Alberto Jaimes-Contreras ${ }^{3}$
}

Recibido: marzo de 2020

Aceptado: octubre de 2020

Para citar este artículo: Hernández-Suárez, C., Méndez-Umaña, J. P., Jaimes-Contreras, L. A. (2021). Memoria de trabajo y habilidades matemáticas en estudiantes de educación básica. Revista Científica, 40(1), 63-73.

https://doi.org/10.14483/23448350.15400

\begin{abstract}
Resumen
Investigaciones recientes han evidenciado que la memoria de trabajo es esencial para el desarrollo de las habilidades matemáticas. El estudio tuvo como objetivo estudiar la mediación de la capacidad matemática con respecto a la memoria de trabajo, en un grupo de estudiantes. El diseño es no experimental, cuantitativo y correlacional; para la evaluación de variables se utilizó la Batería Evaluación Neuropsicológica Infantil, y una prueba sobre habilidades matemáticas en un grupo de estudiantes de educación básica primaria. Los datos analizados indican que se encuentra una correlación significativa entre memoria de trabajo y las habilidades matemáticas en el grupo evaluado. Se puede apreciar que un entrenamiento de la amplitud de memoria de trabajo puede fortalecer significativamente las habilidades matemáticas de los estudiantes.
\end{abstract}

Palabras clave: memoria de trabajo, habilidades matemáticas, procesamiento de información, educación basica.

\begin{abstract}
Recent research has shown that working memory is essential for the development of mathematical skills. The aim of the study was to study the mediation of mathematical ability with respect to working memory in a group of students. The design is nonexperimental, quantitative, and correlational; for the evaluation of variables the Neuropsychological Assessment of Children Battery and a test on mathematical skills in a group of primary school students were used. The data analysed indicate that a significant correlation is found between working memory and mathematical skills in the assessed group. A training of the working memory range can significantly strengthen the students' mathematical skills.
\end{abstract}

1. Universidad Francisco de Paula Santander. Cúcuta, Norte de Santander, Colombia. cesaraugusto@ufps.edu.co

2. Universidad Cooperativa de Colombia, Bogotá, Colombia. juliana.mendez@campusucc.edu.co

3. Escuela Colombiana de Ingeniería Julio Garavito. Bogotá, Colombia. luis.jaimes@escuelaing.edu.co 
Keywords: working memory, mathematical skills, information processing, basic education.

\section{Resumo}

Pesquisas recentes mostraram que a memória de trabaIho é essencial para o desenvolvimento de competências matemáticas. O objectivo do estudo era estudar a mediação da capacidade matemática com respeito à memória de trabalho num grupo de estudantes. A concepção é não experimental, quantitativa e correlacional; para a avaliação das variáveis foi utilizada a Bateria de Avaliação Neuropsicológica Infantil, e um teste sobre competências matemáticas num grupo de alunos do ensino primário. Os dados analisados indicam que é encontrada uma correlação significativa entre a memória de trabalho e as capacidades matemáticas no grupo avaliado. Pode-se apreciar que uma formação da amplitude da memória de trabalho pode reforçar significativamente as capacidades matemáticas dos estudantes.

Palavras-chaves: memória de trabalho, competências matemáticas, processamento de informação, educação básica.

\section{Introducción}

Del resultado del informe PISA 2018, prueba que evaluó a los estudiantes de 15 años para medir qué tan preparados están para entender y enfrentar los retos prácticos de la vida adulta, muestra un panorama educativo poco alentador para Colombia, a pesar del crecimiento que se ha tenido desde el 2006, donde se concluye que los estudiantes en matemáticas obtuvieron un rendimiento menor (391) que la media de la OCDE (481), de los cuales casi $40 \%$ tuvieron un bajo nivel de logro (Echazarra y Schwabe, 2019).

Esta situación plantea retos significativos por asumir y encontrar mecanismos efectivos para aumentar las habilidades de los estudiantes en matemáticas, en especial de básica primaria y en el futuro mejorar los resultados en este tipo de pruebas y elevar la pertinencía del sistema educativo del país a la altura de los mejores.
En este sentido, existen multiples causas para el fracaso o del éxito escolar, tanto en matemáticas como en cualquier otra discipñina, que va desde lo personal hasta lo sociocultural, y que en la mayoría es una mezcla de ambos (Papalia et al., 2009). A nivel psicológico, se pueden mencionar los trastornos del desarroIlo (Willcutt et al., 2013; Reigosa-Crespo et al., 2012), de la inteligencia (Geary et al., 2012), la personalidad (Neuenschwander et al., 2013), el autoconcepto (Marsh y Martin, 2010), y la ansiedad (Karimi y Venkatesan, 2009) entre los mas sobresalientes.

En este sentido, entender la neuropsicología como una perspectiva para comprender los procesos de aprendizaje normal y los trastornos de aprendizaje (López, 2011), implica mejorar los indicadores educativos de los estudiantes. En este marco, se puede afirmar que las diferencias individuales en procesos cognitivos se relacionan con las habilidades matemáticas (Passolunghi y Mammarella, 2012; Lefevre et al., 2013; Vukovic y Lesaux, 2013), como las funciones ejecutivas, en este caso, la memoria de trabajo (MT).

Por otra parte, cuando se hace al aprendizaje de las matemáticas es necesario tener en cuenta los procesos que se llevan a cabo para el aprendizaje de estas (adquisición de conocimientos y procesamiento de información). En este caso es relevante hablar de la MT, teniendo en cuenta que se concibe como un sistema cerebral que proporciona almacenamiento temporal de la información, manteniéndola activa mientras se trabaja con ella, y permitiendo la comprensión de aprendizajes complejos (López, 2014).

De acuerdo con lo anterior, el estudio tiene como finalidad estudiar la mediación de la capacidad matemática con respecto a la MT, en un grupo de estudiantes de educación básica primaria, lo que permite conocer con mas profundidad la problemática descrita con el fin de fortalecer el proceso de aprendizaje a través de programas neuropsicólogicos. 


\section{Memoria de trabajo (MT)}

Etchepareborda y Abad-Mas (2005) señalan que el ser humano en sus primeros años de vida se caracteriza por tener una memoria sensitiva, la cual es la encargada de guardar las primeras sensaciones y las diversas emociones que vive el sujeto; posteriormente aparece la llamada memoria de las conductas que hace referencia a los movimientos que van adquiriendo y, al mismo tiempo se van memorizando, de esta manera el niño adquiere nuevos aprendizajes y se adapta al ambiente que le rodea a diario. Además, consideran que la memoria de conocimiento es la capacidad de guardar datos y evocarlos en el momento en que sea necesario. En síntesis, definen que la memoria es la capacidad de retener y de recordar situaciones vividas del pasado, mediante procesos neurobiológicos de guardar y de recuperar la información y resulta fundamental en el proceso de aprendizaje y en el pensamiento.

El modelo estructural de Atkinson y Shiffrin (1968) considera que la memoria tiene una división de tres niveles. La primera, hace referencia a la memoria inmediata o sensorial (MS), que permite retener los datos o información que recogemos a través de los órganos de los sentidos y que se mantiene por periodos cortos de tiempo. El segundo nivel de memoria, menciona la memoria mediata, a corto plazo (MCP), que se encarga de almacenar y procesar algunos elementos durante un tiempo, a su vez, nos permite contrastar los datos adquiridos facilitando el aprendizaje de tareas de alta complejidad. Finalmente, el modelo incluye una tercera estructura que hace referencia a la memoria diferida o memoria a largo plazo (MLP), es decir, a los conocimientos que hemos adquirido o aprendido.

Baddeley (2003a) menciona que la MT involucra el almacenamiento y la manipulación de la información que se presume que es necesaria para llevar a cabo una amplia gama de actividades cognitivas complejas. El modelo de MT de Baddeley e Hitch (1974) en un inicio consistía en tres componentes o subsistemas funcionales: Ejecutivo central, agenda visoespacial y bucle fonológico; posteriormente, Baddeley propuso la existencia de otro subsistema: El buffer episódico.

Según Delgado-Losada (2015), el estudio de las operaciones realizadas con la información bidireccional recibida revela que en la memoria operativa se llevan a cabo operaciones mentales simultáneas pertenecientes a diferentes contenidos. Unas relacionadas con la información visual (agenda visoespacial: La cual retiene y mantiene activa de forma temporal la visualización del material visual-espacial), y de forma simultánea otras relacionadas con la información auditiva, de carácter verbal, vinculadas al habla (bucle fonológico o lazo articulatorio, el cual realiza una función análoga a la de la agenda visoespacial, pero, en este caso, a través de información de tipo verbal-acústica).

El problema más importante del modelo de MT de Baddeley e Hitch era explicar cómo se relacionaba esta con la MLP, por lo que, posteriormente Baddeley (2000) actualizó el modelo incluyendo un nuevo componente: El buffer episódico. Este consiste en un sistema de almacenamiento temporal con capacidad limitada, capaz de integrar información de varias fuentes incluyendo la agenda visoespacial, el bucle fonológico (uno para el conocimiento espacial y otro para la adquisición del lenguaje) y la MLP.

En síntesis, la MT es la capacidad que empleamos cuando intentamos retener información acerca de algunas situaciones que acaban de suceder o pensamientos inmediatos que acabamos de tener, y que, por medio del razonamiento, damos paso a la resolución mental de un problema o en la toma de una decisión específica en un momento dado (Morgado, 2005).

Un adecuado funcionamiento de los subsistemas de la MT le permite al sujeto adquirir diversos aprendizajes como los fonemas de las palabras, retención de dígitos, entre otros procesos cognitivos complejos. Por lo tanto, cuando el sistema de almacenamiento de la información está bien estructurado y organizado la recuperación de dicha 
información va a depender de la codificación realizada en un primer momento de dicha información (Ballesteros, 1999).

\section{Fundamento neurológico}

Diversas investigaciones han tratado de definir las estructuras cerebrales implicadas en la memoria. Según Morgado (2005), la memoria se ubica en el lóbulo temporal, específicamente en el hipocampo.

Por otra parte, Gordillo (2014) menciona que la MT tiene sus bases neurológicas en el cortex prefrontal, teniendo en cuenta que esta área realiza un rol esencial en las funciones de la MT, y, a su vez, hace parte de una red que une varias áreas, $y$, tiene una influencia puntual, destacando de esta manera que la agenda visoespacial se encuentra relacionada con el lóbulo parietal posterior y el bucle fonolólogico está relacionado con áreas temporales

López (2014) menciona que los estudios con neuroimágenes demuestran que la activación de la corteza prefrontal parece estar relacionadas a funciones de la MT, como el mantenimiento provisional de los resultados intermedios, la planificación y la ordenación temporal de los componentes de las tareas y la comprobación de resultados y la corrección de errores.

De igual forma la participación de la región del lóbulo prefrontal en los circuitos neuronales de las matemáticas, se relacionaría con el almacenamiento y la recuperación necesaria para la solución de actividades matemáticas (López, 2014).

\section{Fundamento educativo}

Alloway (2006) propone que la importancia de la MT en el aula radica, especialmente, en que es importante para aquellas actividades que requieren almacenar la información y procesarla al mismo tiempo. Por lo tanto, es un proceso que requiere de comprensión, ya que, al intervenir diversos procesos psicológicos, puede prestarse a confusión, especialmente en los docentes, con problemas de atención y motivación cercanos al trastorno por déficit de atención e hiperactividad.

Gathercole \& Alloway (2007) proponen que los estudiantes tienen dificultades en la MT, pueden tener dificultades de aprendizaje. Esto se puede explicar debido a que esta memoria proporciona la capacidad de ir integrando información en la memoria a largo plazo al mismo tiempo que se va almacenando y procesando la información a corto plazo. Además, al ser un proceso básico que se necesita para realizar procesos superiores, estos no pueden funcionar adecuadamente (Alloway, 2006) y así el estudiante tendrá una limitada habilidad para realizar actividades en el aula.

Por lo tanto, la MT al ser un proceso que esta relacionado con el aprendizaje, si se entrena, mejora no solo este proceso sino otros dependientes, ya que la intervención educativa, ejerce un efecto positivo en la adquisición de nuevos aprendizajes y a su vez fortalece los procesos académicos de los estudiantes (Alsina y Sáiz, 2004). El entrenamiento de la memoria debe tener en cuenta aspectos como la organización de la información a evocar (la información bien estructurada se codifica con mayor facilidad). De igual forma, es necesaria la comprensión de la información, el conocimiento previo del sujeto y la habilidad para usar las estrategias necesarias que favorecen el proceso de almacenamiento (Etchepareborda y Abad-Mas, 2005).

\section{Habilidades matemáticas básicas}

Geary (2007), evidencia que el ser humano posee unas competencias cuantitativas mensurables básicas implícitas o innatas, que durante la infancia y los años preescolares constituyen la base para el aprendizaje posterior de las matemáticas:

Numerosidad (habilidad de determinar, sin contar y de forma precisa, un máximo de tres o cuatro elementos presentados); ordinalidad (capacidad para detectar cuál de dos conjuntos, de tres o cuatro elementos a lo sumo, es mayor o menor en número); conteo (capacidad para numerar y contar 
pequeños grupos de ítems mediante un sistema no verbal); aritmética simple (sensibilidad para detectar incrementos y decrementos en la cantidad de un conjunto formado por pocos elementos); estimación (valoración de cantidades, magnitudes y tamaños relativos) y geometría (comprensión de la forma y de las relaciones espaciales).

Sobre estas competencias matemáticas biológicas, hay que ir añadiento las competencias o procesos mentales que se dan y son necesarios para desarrollar el pensamiento matemático, que se adquieren con el desarrollo cognitivo. Por ejemplo, de la numerosidad puede surgir evolutivamente como una capacidad numérica.

En este mismo sentido, Orrantia (2006), manifiesta que el desarrollo de las habilidades como el conteo verbal (palabras numéricas y conteo, que incluye los principios de correspondencia uno a uno, de orden estable, de cardinalidad, de abstracción y de irrelevancia) y esquemas protocuantitativos (comparación, incremento-decremento, parte todo) son la base para enfrentarse a la resolución informal de las primeras situaciones problemáticas que incluyen suma y resta.

\section{Relación entre la memoria de trabajo y las habilidades matemáticas}

Ashkenazi et al. (2013), explica que las dificultades en matemáticas se deben a problemas con la memoria de trabajo. Esto se evidencia en múltiples estudios que relacionan la MT con las habilidades matemáticas, lo que establece un campo de investigación en la psicología de la memoria (Alsina \& Sáiz, 2003). Se pueden destacar los trabajos de Rodríguez-Villagra (2015); Alsina (2007), López (2014), Gordillo (2014), Sala-Galindo (2014), entre otros.

Friso-van den Bos et al. (2013) exponen algunos ejemplos de cómo las tres funciones ejecutivas básicas que se producen gracias a la memoria de trabajo, la inhibición, la flexibilidad y la actualización de la información, se relacionan con las matemáticas.

\section{Método}

\section{Enfoque y diseño}

El estudio realizado corresponde a una investigación cuantitativa. El diseño que se utilizó es de tipo no experimental correlacional, que intenta conocer la relación entre la MT y las habilidades matemáticas de los estudiantes de educación básica primaria.

\section{Población y muestra}

La institución educativa objeto de estudio se encuentra ubicada en Suba - Bogotá, Colombia. Es una institución privada, con jornada única, a nivel de preescolar, básica y media académica, tanto para estudiantes regulares como con necesidades educativas especiales. El tipo de muestra es no probabilística, dado que la elección de los elementos no depende de la probabilidad sino de causas relacionada con las características de la investigación. La muestra estuvo conformada por 40 estudiantes de cuarto grado de básica primaria, de ambos géneros con edades comprendidas entre 9 y 11 años, sin trastornos de aprendizaje en matemáticas.

\section{Variables e instrumentos}

Las variables estudiadas son: Capacidad de la MT y habilidades matemáticas. A continuación, se describe el tipo de instrumento a utilizar para la medición de estas variables.

- Variable 1, Capacidad de la MT: Se busca estudiar la MT de un determinado grupo de estudiantes, la medida de la variable es determinada con la Evaluación Neuropsicológica Infantil ENI de (Matute et al., 2007), por medio de dos subtets dígitos inversos y dígitos directos, con una puntuación numérica. La subprueba de dígitos es una tarea de MT, tanto de conservación de la información (dígitos directos), como de manipulación interna de la información (dígitos inversos) (ver tabla 1). La aplicación se 
realiza de forma individual y en esta el estudiante en la prueba de dígitos de orden directo debe repetir un listado de números en el mismo orden en que el entrevistador los presenta; en la segunda parte que hace referencia a los dígitos de orden inverso, el estudiante debe repetir los números en orden inverso a como los ha dicho el entrevistador. La ficha técnica de la batería se ubica en una compilación sobre neuropsicología infantil en lo concerniente a pruebas neuropsicológicas de Matute et al. (2010).
- Variable 2, habilidades matemáticas: Se describe las habilidades matemáticas evaluado por medio de una prueba con item sobre habilidades simples y complejas (Geary, 2007) (ver tabla 2).

Las puntuaciones de la prueba son de tipo numérico. Para ello se utiliza el sistema de valoración la escala numérica de veinte (20) a cincueta (50) de la institución educativa objeto de estudio:

Tabla 1. Características Capacidad de la memoria de trabajo.

\begin{tabular}{lll}
\hline \multicolumn{1}{c}{ Tarea } & \multicolumn{1}{c}{ Subprueba } & \multicolumn{1}{c}{ Objeto } \\
& & $\begin{array}{l}\text { Debe repetir } \\
\text { listado de núm }\end{array}$ \\
$\begin{array}{ll}\text { Conservación de } \\
\text { la información }\end{array}$ & $\begin{array}{l}\text { Retención de dígitos } \\
\text { en progresión (Dígitos } \\
\text { directos) }\end{array}$ & $\begin{array}{l}\text { en mismo } \\
\text { orden en que } \\
\text { entrevistador }\end{array}$
\end{tabular}

Manipulación interna de la información
Debe repetir un listado de números en el mismo orden en que el entrevistador los presenta
El estudiante debe repetir los números en orden inverso a como los ha dicho el entrevistador
Procedimiento

Se menciona un dígito por segundo. Si logra repetir los tres dígitos iniciales, se pasa a la serie de cuatro dígitos. Si repite la serie de cuatro, se pasa a la serie de cinco y así sucesivamente. La serie se presenta una sola vez. Si el sujeto solicita que se la repitan, o bien se equivoca, se pasa a la segunda serie que contiene el mismo número de dígitos. Si fracasa nuevamente, se suspende la prueba. La puntuación obtenida es equivalente al número máximo de dígitos que el sujeto logre repetir en esta prueba, ya sea en el primero o en el segundo ensayo. La puntuación máxima posible es de 9 puntos.

Se menciona un dígito por segundo. Si logra repetir en forma inversa los dos dígitos iniciales, se pasa a la serie de tres dígitos. Si repite la serie de tres, se pasa a la serie de cuatro y así sucesivamente. La serie se presenta una sola vez. Si el sujeto solicita que se la repitan, o bien se equivoca, se pasa a la segunda serie que contiene el mismo número de dígitos. Si fracasa nuevamente, se suspende la prueba.

La puntuación obtenida es equivalente al número máximo de dígitos que el sujeto logre repetir en esta prueba, ya sea en el primero o en el segundo ensayo. La puntuación máxima posible es de 8 puntos.

Fuente: elaboración propia de los autores.

Tabla 2. Características Habilidades matemáticas.

\begin{tabular}{llll}
\hline Habilidades & Operación & Capacidad & Items \\
& $\begin{array}{l}\text { Operaciones numéricas básicas } \\
\text { Habilidades simples }\end{array}$ & $\begin{array}{l}\text { Haçer sumas y restas } \\
\text { Ordenar series de numeros de }\end{array}$ & $\begin{array}{l}12 \text { operaciones } \\
\text { mayor a menor }\end{array}$ \\
& Producción numes para ordenar \\
Habilidades complejas & Problemas aritmeticos & $\begin{array}{l}\text { Traducir los numeros de un tipo } \\
\text { de representación a otra }\end{array}$ & 8 producciones \\
& & $\begin{array}{l}\text { Resolver problemas aritméti- } \\
\text { cos basados en operaciones } \\
\text { aritméticas. }\end{array}$ & 5 problemas. \\
\hline
\end{tabular}

Fuente: elaboración propia de los autores de acuerdo con Geary (2007). 
Tabla 3. Escala de valoración institucional.

\section{Escala}

Superior $46-50$

Alto 40-45

Básico 35-39

Bajo 20- 34

\section{Descripción}

Generalmente demuestra con suficiencia la competencia en variadas y complejas situaciones y los resultados superan lo esperado.

Muchas veces demuestra la competencia en diferentes situaciones complejas y los resultados cumplen lo esperado. Conoce algunos fundamentos conceptuales que le permite desempeñarse adecuadamente.

Demuestra la competencia en algunas situaciones de mediana complejidad y los resultados alcanzan lo mínimo esperado. Desconoce los fundamentos conceptuales que le permiten desempeñarse adecuadamente, pero los aplica de forma intuitiva.

Rara vez demuestra la competencia en situaciones de baja complejidad y los resultados no alcanzan lo mínimo esperado. No conoce los fundamentos conceptuales que le permiten hacerlo adecuadamente.

Fuente: elaboración propia de los autores.

\section{Hipotesis}

1. $\mathrm{H}_{0}$ : no existe relación entre la MT y las habilidades matemáticas.

2. $\mathrm{H}_{1}$ : se espera encontrar una relación positiva entre la MT y las habilidades matemáticas.

Las variables de MT y las habilidades matemáticas están relacionadas.

\section{Procedimiento}

El procedimiento realizado se dio por diversas fases. La primera fue dar a conocer el estudio a las directivas de la institución con el fin de solicitar la población para la aplicación de las subpruebas. Posteriormente, se dio a conocer a los docentes - directores de grupo, con el objeto de solicitar la población en un espacio en que ellos consideraran pertinente y al docente de Matemáticas, las notas respectivas de los estudiantes obtenidas. Teniendo seleccionado el grupo de estudio (cuarto de primaria), se procedió a hacer una reunión con padres de familia. Se les dio a conocer la investigación y el consentimiento informado para hacer parte del estudio propuesto. La respuesta por parte de los padres de familia fue positiva, aceptando la participación de sus hijos.
Teniendo en cuenta los horarios de clases se procedió a llamar a cada uno de los participantes, aplicando primero la subprueba de dígitos de orden directo y posteriormente la de dígitos de orden inverso, con un estimado de aplicación de 15 minutos por estudiante. Para finalizar se realizó un análisis estadístico correlacional para contrastar la hipótesis propuesta.

\section{Análisis de datos}

Una vez obtenidos los datos de cada variable de estudio, se llevó a cabo el análisis estadístico por medio de correlaciones (Pearson) para analizar el contraste en de hipótesis en cuanto a la MT y las habilidades matemáticas. Para el análisis se utilizo el complemento del MS Excel denominado EZAnalyze.

\section{Resultados}

A continuación, en la tabla 4 se presentan los estadísticos descriptivos de las variables.

En la tabla 5 se presenta la correlación de la amplitud de memoria y las habilidades matemáticas. Dicha correlación (Pearson) se obtuvo a través del Excel EZAnalyze; con esto se buscó analizar la amplitud de memoria de los estudiantes al aplicar la prueba de dígitos directos y las puntuaciones obtenidas en la prueba de habilidades matemáticas: 
Tabla 4. Estadísticos descriptivos de las variables experimentales $(N=40)$.

\begin{tabular}{ccccc}
\hline Variables & Media & D.T & Min & Max \\
Dígitos directos & 5,1 & 1,1 & 3,0 & 7,0 \\
Dígitos inversos & 4,3 & 1,0 & 2,0 & 7,0 \\
Prueba de habilidad matemática & 34,0 & 7,2 & 20,0 & 50,0 \\
\hline
\end{tabular}

Fuente: elaboración propia de los autores.

Tabla 5. Correlación amplitud de memoria y las habilidades matemáticas.

\begin{tabular}{ccc}
\hline & Habilidades matemáticas & \\
& $\mathrm{R}$ & $\mathrm{P}$ \\
Amplitud de memoria & 0,39 & 0,01 \\
\hline
\end{tabular}

Fuente: elaboración propia de los autores.

Tabla 6. Correlación capacidad de MT y las habilidades matemáticas

\begin{tabular}{ccc}
\hline & Habilidades matemáticas & \\
Capacidad de MT & $\mathrm{R}$ & $\mathrm{P}$ \\
& 0,41 & 0,01 \\
\hline
\end{tabular}

Fuente: elaboración propia de los autores.

Teniendo en cuenta los resultados obtenidos, se puede encontrar que existe una correlación significativa entre la amplitud de memoria y las habilidades matemáticas. Teniendo en cuenta que el valor estadístico $(p)$ se encuentra por debajo de 0,05 para este caso es de 0,01 y el coeficiente de correlación ( $r$ ) para estas dos variables es de 0,39, lo que indica una correlación significativa entre ellas, lo cual evidencia que una mayor amplitud de memoria involucra resultados positivos en la prueba de habilidades matemáticas.

Además, de los datos obtenidos al aplicar la prueba de dígitos inversos (tabla 6), se concluye que hay una correlación estadísticamente significativa entre las variables capacidad de MT y la prueba de habilidades matemáticas, teniendo en cuenta que la probabilidad asociada al análisis $(p)$ se encuentra por debajo de 0,05, en este caso es el de 0,01. Por otra parte, el coeficiente de correlación ( $r$ ) para las variables es de 0,41, lo cual también indica que a mayor capacidad en la MT hay mejores resultados en la prueba de habilidades matemáticas.

\section{Discusión}

El propósito de esta investigación fue estudiar la relación de la MT y las habilidades matemáticas en estudiantes, por lo que la hipótesis de investigación planteada se rechaza para aceptar la hipótesis alternativa, ya que se ha encontrado que la MT está significativamente relacionada con el desarrollo de las habilidades matemáticas en la población objeto de estudio.

Esta relación significativa se ha encontrado en estudios realizados de MT y el progreso académico en matemáticas en estudios como el de Alsina y Sáiz (2003), en el cual mencionan que una baja amplitud en el proceso de MT suele influir en un bajo rendimiento de las matemáticas. Es decir, puntuación baja en las medidas de la MT suelen tener un rendimiento por debajo de los patrones 
esperados en diferentes áreas académicas (García et al., 2018).

También es pertinente lo planteado por López (2013b; 2014) quien evaluó el desempeño de cada variable como bucle fonológico, agenda visoespacial, ejecutivo central y su conocimiento en el cálculo aritmético y concluye que hay una relación entre el desarrollo progresivo de la amplitud de MT y los desempeños de matemáticas a través del tiempo.

Ademas, se encuentra a Gordillo (2014) quien en su estudio acerca de la relación de la MT, lateralidad y rendimiento escolar en matemáticas, encontró una relación significativa entre las variables MT y rendimiento de las matemáticas en estudiantes de segundo grado de primaria.

Lo expuesto anteriormente, significa que entre más escolaridad aumenta el número de retención de dígitos (Ardila y Ostrosky, 2012). En este sentido, es importante la estimulación y fortalecimento en los primeros años escolares, de las estructuras funcionales como la MT, ampliando de esta forma la posibilidad de optimizar las capacidades de los estudiantes (Sala-Galindo, 2014), lo que muestra la importancia que tienen las funciones ejecutivas en los procesos de aprendizaje (Fonseca et al., 2016).

Finalmente, la afectación que tenga un estudiante en su rendimiento escolar no se puede generalizar, por el contrario, esta debe ser vista en sus particularidades e incluir tanto factores como la atención y la concentración, así como las condiciones de estudio del estudiante, las características del aula y, en general, la relación propia de este con su entorno. Por lo que es necesario la elaboración de programas de intervención que mejoren el progreso académico mediante el diseño de estrategias aplicables a cualquier asignatura (Parra y de la Peña, 2017), en este caso la de Matemáticas.

\section{Conclusión}

Las habilidades matemáticas son el centro de lo que se evalúa en matemáticas, y, por tanto, es un elemento que influye en el rendimiento académico de los estudiantes. Por lo que es lógico que, si la MT predice estadísticamente las habilidades matemáticas, también debe estar asocidada con el rendimiento académico. Sin embargo, aunque el estudio demostró la incidencia el desarrollo de las habilidades matemáticas de los estudiantes, en la población objetivo no posibilita la realización de generalizaciones acerca de la relación con la MT, debido a que el tamaño de la muestra es pequeño y a que no se evalúan todas las habilidades matemáticas de acuerdo con el marco propuesto, sino que se obtienen resultados parciales que permitirán determinar con mayor precisión la relación entre estas variables en un estudio futuro que incluya un mayor número de estudiantes inclusive de otros grados e instituciones así como habilidades matemáticas complejas, para dar cuenta de estas habilidades de los estudiantes afectan el proceso de aprendizaje del área de Matemáticas.

\section{Referencias}

Alloway, T. (2006). How does working memory work in the classroom? Educational Research and Reviews, 1(4),134-139. https://doi. org/10.5897/err.9000188

Alsina, A. (2007). ¿Por qué algunos niños tienen dificultades para calcular? Una aproximación desde el estudio de la memoria humana. Revista Latinoamericana de Investigación en Matemática Educativa, 10(3), 315-333.

Alsina, A., Sáiz, D. (2003) ¿Es posible entrenar la memoria de trabajo?: un programa para niños de 7-8 años. Infancia y Aprendizaje, 27(3), 275-287.

Ardila, A., Ostrosky, F. (2012). Guía para el diagnóstico neuropsicológico. http://ineuro.cucba. udg.mx/libros/bv guia para el diagnostico neuropsicologico.pdf

Atkinson, R., Shiffrin, R. (1968). Human memory: a proposer system and is control processes. En E. W. K. Spence y J. T. Spence (eds.), The Psychology of learning and motivation: Advance in Research and Theory (pp. 89-195). Academic press. 
Baddeley, A. (2000). The episodic buffer: a new component of working memory? Trends in Cognitive Sciences, 4 (11), 417-423. http://doi. org/10.1016/S1364-6613(00)01538-2

Baddeley, A. (2003). Working memory: Looking back and looking forward. Nature Reviews Neuroscience, 4, 829-839

Baddeley, A., Hitch, G. (1974). Memoria en funcionamiento. En M. V. Sebastián (ed), Lecturas de psicología de la memoria (pp. 471-485). Alianza Editorial.

Ballesteros, S. (1999). Memoria humana: investigación y teoría. Psicothema, 11(4), 705-723. http://www.psicothema.com/pdf/323.pdf

Delgado-Losada, M. (2015). Memoria. En fundamentos de psicología para ciencias sociales y de la salud. Ed. Panamericana.

Echazarra, A., Schwabe, M. (2019). Colombia - Country Note - PISA 2018 Results. OECD Publishing. https://www.oecd.org/pisa/publications/PISA2018 CN COL ESP.pdf

Etchepareborda, M., Abad-Mas, L. (2005). Memoria de trabajo en los procesos básicos del aprendizaje. Revista de neurología, 40(1), S79-S83. https://doi.org/10.33588/rn.40S01.2005078

Fonseca, G., Rodríguez, L., Parra, J. (2016). Relación entre funciones ejecutivas y rendimiento académico por asignaturas en escolares de 6 a 12 años. Hacia promoción de la salud, 21(2), 41-58. http://doi.org/10.17151/ hpsal.2016.21.2.4

Friso-van den Bos, I., Van der Ven, S., Kroesbergen, E., Van Luit, J. (2013). Working memory and mathematics in primary school children: A meta-analysis. Educational Research Review, 10, 29-44. http://doi.org/10.1016/j. edurev.2013.05.003

Gathercole, S., y Alloway, T. (2007). Understanding working memory: a classroom guide. Harcourt Assessment

Geary, D. (2007). An evolutionary perspective on learning disability in mathematics. Developmental Neuropsychology, 32(1), 471-519. https://doi.org/10.1080/87565640701360924
Geary, D., Hoard, M., Nugent, L. (2012). Independent contributions of the central executive, intelligence, and in-class attentive behavior to developmental change in the strategies used to solve addition problems. Journal of Experimental Child Psychology, 113(1), 49-65. https://doi.org/10.1016/j. jecp.2012.03.003

Gordillo, B. (2014). Influencia de la memoria de trabajo y lateralidad en el rendimiento académico en matemáticas de niños de Segundo de primaria (tesis fin de máster) Universidad Internacional de La Rioja, España. http://reunir. unir.net/bitstream/handle/123456789/2393/ gordillo $\% 20$ ballen.pdf? sequence $=1$

Karimi, A., Venkatesan, S. (2009). Mathematics Anxiety, Mathematics Performance and Academic Hardiness in High School Students. International Journal of Educational Sciences, 1(1),33-37. https://doi.org/10.1080/09751122 .2009 .11889973

Lefevre, J., et al. (2013). The role of executive attention in the acquisition of mathematical skills for children in Grades 2 through 4. Journal of Experimental Child Psychology, 114(2), 243-261. https://doi.org/10.1016/j.jecp.2012.10.005

López, M. (2011). Memoria de trabajo y aprendizaje: de la neuropsicología. Cuadernos de $\mathrm{Neu}$ ropsicología., 5(1), 25-47. http://www.cnps.cl/ index.php/cnps/article/view/115

López, M. (2013). Rendimiento académico: su relación con la memoria de trabajo. Revista Electrónica Actualidades Investigativas en Educación, 13(3), 1-19. http://doi.org/10.15517/ aie.v13i3.12042

López, M. (2014). Desarrollo de la memoria de trabajo y desempeño en cálculo aritmético: un estudio longitudinal en niños. Electronic Journal of Research in Educational Psychology, 12(1), 171-190. https://www.redalyc.org/ pdf/4396/439642487003.pdf

Marsh, H., Martin, A. (2011). Academic self-concept and academic achievement: relations and causal ordering. The British Journal of 
Educational Psychology, 81(1), 59-77. https:// doi.org/10.1348/000709910X503501

Matute, E., Rosselli, M., Chamorro, Y., Orozco, J. (2010). Pruebas neuropsicológicas infantiles en español. En M. Rosselli, E. Matute y A. Ardila (eds.), Neuropsicología del desarrollo infantil (pp. 119-136). El Manual Moderno.

Méndez, J. (2016). Relación de la memoria de trabajo y el rendimiento de las matemáticas en niños de cuarto grado de primaria (tesis fin de máster) Universidad Internacional de La Rioja, España. https://reunir.unir.net/bitstream/handle/123456789/4873/MENDEZ\%20OMAÑA \% 2 C \% 20 JULIANA \% 20 PAOLA. pdf? sequence $=1$ \&isAllowed $=y$

Morgado, B. (2005). Psicobiología del Aprendizaje y la Memoria. CIC (Cuadernos de Información y Comunicación), 10, 221-233. https:// revistas.ucm.es/index.php/CIYC/article/view/ CIYC0505110221A

Neuenschwander, R., Cimeli, P., Röthlisberger, M., Roebers, C. (2013). Personality factors in elementary school children: Contributions to academic performance over and above executive functions? Learning and Individual Differences, 25, 118-125. https://doi.org/10.1016/j. lindif.2012.12.006

Papalia, D., Wendkos, S. y Duskin, O. (2009). Desarrollo humano. De la Infancia a la adolescencia. McGraw Hill Interamericana

Parra, N., dela Peña, I. (2017). Atención y memoria en estudiantes con bajo rendimiento académico. Un estudio exploratorio. ReiDoCrea, 6, 74-83. http://hdl.handle.net/10481/45029

Passolunghi, M., Mammarella, I. (2012). Selective spatial working memory impairment in a group of children with mathematics learning disabilities and poor problem-solving skills. Journal of Learning Disabilities, 45(4), 341-350. https:// doi.org/10.1177/0022219411400746

Reigosa-Crespo, V., et al. (2012). Basic numerical capacities and prevalence of developmental dyscalculia: the Havana Survey. Developmental Psychology, 48(1), 123-35. https://doi. org/10.1037/a0025356

Rodríguez-Villagra, O. (2015). Modelos de variables latentes, modelación cognitiva y memoria de trabajo: un punto de encuentro. Actualidades en psicología: medición y psicometría, 29(119), 43-62. http://dx.doi.org/10.15517/ ap.v29i119.18850

Rosselli, M. et al. (2004). Evaluación Neuropsicológica Infantil (ENI): batería para la evaluación de niños entre 5 y 16 años de edad. Estudio normativo colombiano. Revista de Neurología, 38(8), 720-731. http://doi.org/10.33588/ rn.3808.2003400

Sala-Galindo, A. (2014). Memoria de trabajo, capacidades matemáticas y rendimiento académico en alumnado de primaria (tesis fin de máster). Universidad Internacional de La Rioja, España. https://reunir.unir.net/bitstream/handle/123456789/2993/AnaPilar Sala_Galindo. pdf? sequence $=3$ \&isAllowed $=y$

Vukovic, R., Lesaux, N. (2013). The language of mathematics: investigating the ways language counts for children's mathematical development. Journal of Experimental Child Psychology, 115(2), 227-244. https://doi.org/10.1016/j. jecp.2013.02.002

Willcutt, E. et al. (2013). Comorbidity between reading disability and math disability: concurrent psychopathology, functional impairment, and neuropsychological functioning. Journal of Learning Disabilities, 46(6), 500-16. https:// doi.org/10.1177/0022219413477476 